\title{
Nonlinear System Identification: Prediction Error Method vs Neural Network
}

\author{
Jinming Sun \\ University of Bremen \\ Bremen, Germany \\ jinming@uni-bremen.de
}

\author{
Yanqiu Huang \\ Pervasive Systems \\ University of Twente \\ Enschede, The Netherlands \\ yanqiu.huang@utwente.nl
}

\author{
Wanli Yu \\ ITEM \\ University of Bremen \\ Bremen, Germany \\ wyu@item.uni-bremen.de
}

\author{
Alberto Garcia-Ortiz \\ ITEM \\ University of Bremen \\ Bremen, Germany \\ agarcia@item.uni-bremen.de
}

\begin{abstract}
System identification has been used in various domains for analyzing system properties and carrying out filtering, prediction and automatic control. Prediction error method (PEM) is one of the classic methods to estimate system parameters and exploit dynamical structure of the studied system; while neural network (NN) is favorable for black-box systems with unknown structures. As the popularity of Internet of Things (IoT) and Cyber-physical systems (CPS) increases, the identification tasks are moving more towards resource-constrained devices. Accordingly, some studies incorporate system prior knowledge into $\mathrm{NN}$ to improve its efficiency. However, it is unclear whether the adapted NN outperforms the classic PEM.

This paper provides a fair comparison between two techniques in terms of estimation accuracy and speed on several common nonlinear systems. The results indicate that $\mathrm{NN}$ is wider applicable and accurate, but more expensive from computational perspective; whereas PEM is more lightweight, but has limitations when the system input has frequent abrupt changes.

Index Terms-system identification, nonlinear system, parameter estimation, neural network, prediction error, time-varying system
\end{abstract}

\section{INTRODUCTION}

System identification is a technique of dynamical modeling based on observed data. It has been applied in the fields of engineering, economics, statistics and physical sciences. And the significance as well as the challenge of nonlinear system estimation are well recognized [1]. For resource-constrained devices in IoT and wireless sensor network (WSN), not only the precision, but the less complexity of a method is extremely desired. For example, OpenMote-CC2538, a leading computing and communication device, has only $32 \mathrm{MHz}$ clock and $32 \mathrm{kB}$ RAM [2], which is difficult to carry out a heavy algorithm.

One of the modeling approaches, state-space equation, has become dominating [3] [4] since Kalman's(1960) work on prediction and control, especially with its insights into physical mechanism of a system. Based on Kalman predictor, a prediction-error method (PEM) [5] is able to do online or recursive estimation with excellent asymptotic properties, due to its close kinship with least squares method and maximum likelihood method [6]. And the system is directly modeled into discrete time [7], which is the most common technique for time-invariant or time-varying linear and nonlinear systems.
Artificial neural network (NN), a generic black-box structure prepared to describe any nonlinear dynamics, grows attraction in recent years with great publicly accessibility. It is, however, quite expensive from computational perspective, even with modern computer power. A lot of researches have been conducted to improve the efficiency of NN since the early demonstration of its usage on nonlinear system identification and control [8]. There was a suggestion to first build a linear model for the system and then use $\mathrm{NN}$ to capture nonlinearity in the residual [9]. Anyway, the model training can only be done offline, i.e., based on the whole available data set. And the sufficiency and speed are highly affected by the network size [10]. Incorporating physical insight can speed up the learning and require fewer samples [11]. There were also works showing that directly identifying a model in continuous time has advantages over in discrete time [12].

More recently, a NN method based on state-space model was proposed in [13]. It contains prior knowledge of system structure and is trained over mini-batches. This significantly speeds up the training process yet retaining the accuracy. However, it is unclear how's this algorithm efficiency comparing to conventional PEM.

The goal of this paper is to investigate the performance of KF-based PEM and state-space based NN on nonlinear systems. It will answer the following questions:

- Which method is more suitable for IoT and WSN devices?

- How are their applicability to different systems?

We compared the algorithms' accuracy and speed on three frequently studied nonlinear systems: a Cascaded Tanks System (CTS) [14] [15], a RLC circuit with nonlinear inductor [13] and an Electro-Mechanical Positioning System (EMPS) [16]. The system schematic pictures are in Fig. 1, and further details are mentioned in Section IV. The rest of this paper is structured as follows: Section II states the nonlinear system identification problem and metrics of accuracy; the brief descriptions of two methods are in Section III; Section IV gives the comparison with experimental analysis; and the summary and possible future research are presented in Section V. 




(a) CTS

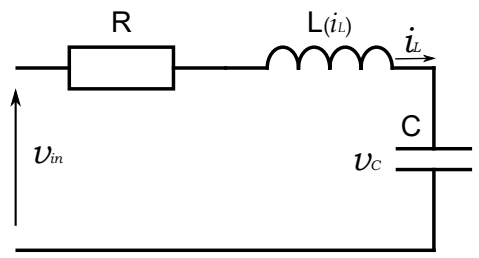

(b) RLC

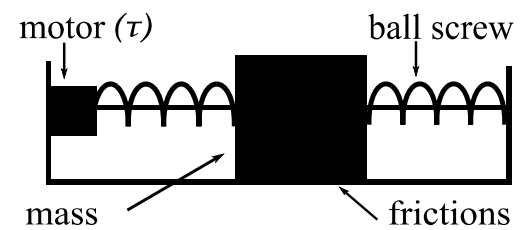

(c) EMPS

Fig. 1. Three nonlinear systems: (a) Cascaded tanks system [14]; (b) a nonlinear RLC circuit where the inductance $L\left(i_{L}\right)$ has nonlinear dependence on the current $i_{L}$ [13]; (c) Electro-mechanical positioning system [16].

\section{PROBLEM STATEMENT}

Typically, a nonlinear system can be modeled in state space form (1), where $x(t)$ denotes the system states and all the unknown parameters are represented in a vector $\theta$.

$$
\begin{aligned}
& \dot{x}(t)=f\left(x(t), u(t), \theta_{1}\right) \\
& y(t)=h\left(x(t), u(t), \theta_{2}\right)
\end{aligned}
$$

The gathered data set $\mathcal{D}$ contains observations of the system inputs $\mathcal{U}=\{u(t), u(t-1), \cdots, u(0)\}$ and outputs $\mathcal{Y}=$ $\{y(t), y(t-1), \cdots, y(0)\}$. The identification task is to find an optimal $\theta$ for the model with given $\mathcal{D}$.

The commonly used metrics for model inference accuracy is the coefficient of determination:

$$
R^{2}=1-\frac{\sum_{k=0}^{N-1}\left(y_{k}-y_{k}^{\text {estimate }}\right)^{2}}{\sum_{k=0}^{N-1}\left(y_{k}-y^{\text {mean }}\right)^{2}}
$$

where $y_{k}$ is the measurement at time $k ; y_{k}^{\text {estimate }}$ is the estimated value at time $k$; and $y^{\text {mean }}$ is the mean of observations.

In this paper, we estimate systems in form (1) and use $R^{2}$ to assess the obtained model.

\section{METHODOLOGY}

This section introduces the ideas of PEM [7] and NN [13].

\section{A. Prediction Error Method (PEM)}

In PEM, the current parameter estimate $\hat{\theta}(t)$ is calculated via the previous estimate $\hat{\theta}(t-1)$ :

$$
\hat{\theta}(t)=\hat{\theta}(t-1)+P(t-1) \psi(t-1) e(t)
$$

where $P(t-1)$ is the algorithm gain, $P(t-1)=P(t-2)-$ $\frac{P(t-2) \psi(t-1) \psi(t-1)^{T} P(t-2)}{I+\psi(t-1)^{T} P(t-2) \psi(t-1)} ; \psi(t-1)$ is a regression vector, $\psi(t-1)=\left.\frac{d \hat{y}(t, \theta)}{d \theta}\right|_{\theta=\hat{\theta}(t-1)}$; and $e(t)$ is the prediction error, $e(t)=y(t)-\hat{y}(t)$.

The state space model uses a canonical form of observability in order to estimate state variables and reduce calculation effort. The optimal objective is to minimize the mean squared errors of prediction:

$$
V_{N}(\theta)=\frac{1}{N} \sum_{t=1}^{N}(y(t)-\hat{y}(t))^{T}(y(t)-\hat{y}(t))
$$

\section{B. Neural Network (NN)}

We focus on feed-forward neural network for our identification tasks. A physics informed NN model (5) is more computational efficient [17], since some parameters are fixed during training. The neural network $\mathcal{N}_{f}$ are trained over subsequences, which are extracted from data set $\mathcal{D}$. So, it can exploit parallel computing, because all the subsequences can be estimated simultaneously.

$$
\begin{aligned}
\dot{x}(t) & =\mathcal{N}_{f}(x(t), u(t), \theta), \quad x(0)=x_{0} \\
y & =g(x)
\end{aligned}
$$

The training objective is to minimize an overall loss consisting of fitting cost and a regularization term used to optimize initial conditions:

$$
J_{\text {total }}(\theta, \bar{X})=J_{f i t}(\theta, \bar{X})+\alpha J_{\text {reg }}(\theta, \bar{X})
$$

where

$$
\begin{aligned}
& J_{f i t}(\theta, \bar{X})=\frac{1}{q m} \sum_{j=0}^{q-1} \sum_{h=0}^{m-1}\left\|\hat{y}_{j, h}(\theta, \bar{X})-y_{j, h}\right\|^{2} \\
& J_{\text {reg }}(\theta, \bar{X})=\frac{1}{q m} \sum_{j=0}^{q-1} \sum_{h=0}^{m-1}\left\|\hat{x}_{j, h}(\theta, \bar{X})-\bar{x}_{j, h}(\bar{X})\right\|^{2}
\end{aligned}
$$

and $\bar{x} \in \bar{X}$ is the subsequence initial value; $q$ is the number of subsequences; $m$ is the length of a subsequence; and regularization weight $\alpha>0$.

\section{EXPERIMENT ANALYSIS}

This section illustrates the performance comparison between PEM and NN using real world data ${ }^{1}$ on three often studied nonlinear systems shown in Fig. 1. All the computations are carried out on a PC with Intel i5-7200U@2.70 GHz and 8GB RAM.

\section{A. Experiment Setup}

With system structural information, model (1) can take format of (8) for CTS, where $x$ is the water levels in tanks; input $u$ is voltage of the pump; the lower tank water level is

\footnotetext{
${ }^{1}$ http://www.nonlinearbenchmark.org
} 
measured as output $y$, and $k_{1} \sim k_{4}$ denote system parameters.

$$
\begin{aligned}
{\left[\begin{array}{c}
\dot{x_{1}} \\
\dot{x_{2}}
\end{array}\right] } & =\left[\begin{array}{c}
-k_{1} \sqrt{x_{1}} \\
k_{2} \sqrt{x_{1}}-k_{3} \sqrt{x_{2}}
\end{array}\right]+\left[\begin{array}{c}
k_{4} u \\
0
\end{array}\right] \\
y & =x_{2}
\end{aligned}
$$

RLC model is in (9), where input $v_{i n}(V)$ is a filtered white noise; measurement $v_{C}(V)$ is the capacitor voltage; $i_{L}(A)$ is the inductor current. And inductance $L$ depends nonlinearly on $i_{L}: L\left(i_{L}\right)=L_{0}\left[0.9\left(\frac{1}{\pi} \arctan \left(-5\left(\left|i_{L}\right|-5\right)+0.5\right)+0.1\right)\right]$.

$$
\begin{aligned}
{\left[\begin{array}{c}
\dot{v_{C}} \\
\dot{i_{L}}
\end{array}\right] } & =\left[\begin{array}{cc}
0 & \frac{1}{C} \\
\frac{-1}{L\left(i_{L}\right)} & \frac{-R}{L\left(i_{L}\right)}
\end{array}\right]\left[\begin{array}{c}
v_{C} \\
i_{L}
\end{array}\right]+\left[\begin{array}{c}
0 \\
\frac{1}{L\left(i_{L}\right)}
\end{array}\right] v_{i n} \\
y & =v_{C}
\end{aligned}
$$

EMPS model is in (10), where motor force $\tau(N)$ is the input; output is joint position $p(m) ; v$ is the joint velocity; $M(\mathrm{~kg})$ is the joint mass; $f_{v}(\mathrm{Ns} / \mathrm{m})$ is dynamic friction coefficient, and $F_{c}(N)$ is the static friction.

$$
\begin{aligned}
{\left[\begin{array}{c}
\dot{p} \\
\dot{v}
\end{array}\right] } & =\left[\begin{array}{cc}
0 & 1 \\
0 & -\frac{f_{v}}{M}
\end{array}\right]\left[\begin{array}{l}
p \\
v
\end{array}\right]+\left[\begin{array}{c}
0 \\
-\frac{1}{M}
\end{array}\right] \tau+\left[\begin{array}{c}
0 \\
-\frac{1}{M}
\end{array}\right] F_{c}(v) \\
y & =p
\end{aligned}
$$

The collected training data set $\mathcal{D}$ contains 1024,10000 and 4968 pairs of input-output samples for CTS, RLC, and EMPS, respectively; and test data have the same size, but different samples. The NN models use ReLU activation, and have one hidden layer with 100, 64, and 64 linear neurons in CTS, RLC and EMPS, respectively.

\section{B. Numerical Analysis}

Training cost relates to model structure complexity, training data size, training iterations and etc.; whereas inference cost depends on the model complexity (i.e., the number of arithmetic operations). In our study, they are reflected through running time. We first use training data to obtain a system model, then feed test data to this model. And its inferences are used in (2) to decide the accuracy of obtained model.

The inference comparisons in terms of accuracy are illustrated in Fig. 2. NN uses the entire training data and the number of iterations can be designed; while iterations in recursive PEM are the number of used samples. In order to get well trained models, NN works 10000 iterations and PEM uses full size of training data mentioned above. It can be seen from Fig. 2a, for CTS, PEM model is more accurate. The predictions of PEM fit much better than NN. Fig. 2b depicts the results comparison in RLC system. Obviously, PEM still works quite well in terms of accuracy, although its precision is slightly worse than NN. This results from the fact that PEM estimates cannot perfectly fit the peaks of the measurements as NN does. For EMPS, Fig. 2c shows that NN performs much better than PEM. It indicates PEM cannot cope with frequently abrupt-changing input signal: its estimates are affected much by the input data. This can result from PEM's linear structure trying to approximate a system with input-output signals of different nonlinearity. In contrast, $\mathrm{NN}$ can deal better with data of highly different frequencies, due to the benefit of its large generic framework.

The training speed comparison is in Fig. 3a. By tuning the iterations in $\mathrm{NN}$, training time is changed and models of different training depth are obtained; in PEM, varying amounts of training data are used. It can be seen that the accuracy of NN inference depends highly on training time; whereas PEM needs much less time to achieve a good $R^{2}$. For instance, to reach $R^{2}$ of 0.98 , in CTS, PEM uses $0.02 s$ and NN uses $784.33 s$; in RLC, $0.02 s$ for PEM and 78.34s for NN. Thus, it's worthy to choose PEM model over NN for system CTS and RLC. The $R^{2}$ of PEM in EMPS is less than zero, meaning a wrong model is chosen for this system [18].

And it should be noted that unlike NN needing abundant data, PEM requires very little amount of samples to build a model. As shown in Fig. 3b, less than 50 samples are sufficient to reach model accuracy of 0.98 in CTS and RLC.

Fig. 3c demonstrates the online inference time if we have already offline-trained models at hand. The test data size are 1024, 10000 and 4968 for CTS, RLC and EMPS, respectively. The results indicate that using PEM model is faster in all the systems, because of its less complex model structure.

In summary, to reach a satisfying accuracy, it's more expensive computational-wise of $\mathrm{NN}$ to work on embedded systems. When considering the trade-off, PEM outperforms $\mathrm{NN}$ in a wide range of examples. If the observed signals' changing are highly different, it's then worthy to use NN. Therefore, even with high interest and investment in neural networks, we can not discard conventional methods like PEM.

\section{CONClusion}

Three nonlinear systems are used to demonstrate the performance of KF-predictor based PEM and state-space based NN.

- The amount of calculations in NN may overburden the devices, while PEM is proved to be significantly faster with good accuracy and demands for few amount of data. Therefore, the trade-off between required precision and computational effort in PEM makes it a better choice for many resource-constrained devices.

- NN can handle the system with input-output data of highly different frequencies. Hence, it's worthy to use NN in some systems with frequently abrupt-changing signals.

- The NN model cannot adapt quickly when the system has changed, because pre-collected data set is needed to train the model offline; while PEM can work simultaneously with data being collected online. Thus, PEM is more adaptive in time-varying systems.

There are still works can be further done. After NN model is trained offline, whenever its credibility is not trustworthy for inference anymore, the retraining may be done with PEM to update the model. But for some systems like EMPS, a mapping rule is needed to modify the input-output data to new sets for PEM to function, e.g., moving averages. This way, the best 

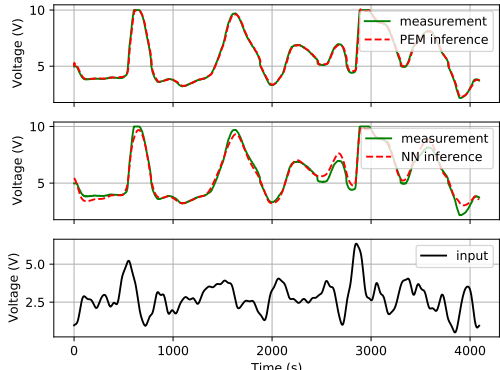

(a) CTS inference.


(b) RLC inference.


(c) EMPS inference.

Fig. 2. Inference results comparison between PEM and NN on three systems. (a) CTS: PEM is more accurate; (b) RLC: PEM works well with accuracy slightly worse than NN; (c) EMPS: NN works well, but PEM is not the right model here.

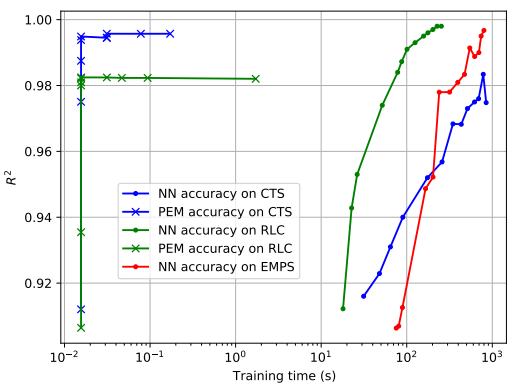

(a)



(b)

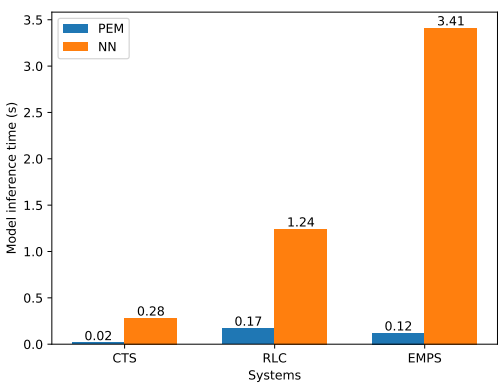

(c)

Fig. 3. Numerical analysis of PEM and NN on three systems. (a) Inference accuracy (coefficient of determination $R^{2}$ ) vs. training time: PEM is obviously faster (not function for EMPS); (b) PEM inference accuracy $R^{2}$ vs. training data size: few samples needed to build a model (not the right model for EMPS); (c) Inference time of PEM and NN models: PEM is faster than NN.

accuracy can be achieved and energy can be spared during retraining.

\section{REFERENCES}

[1] L. Ljung, "Perspectives on system identification," in 17th World Congress The International Federation of Automatic Control, Seoul, Korea, Jul.6-11 2008, pp. 7172-7184.

[2] X. Vilajosana, P. Tuset, T. Watteyne, and K. Pister, "Openmote: opensource prototyping platformfor the industrial IoT," in 7th EAI International Conference on Ad Hoc Networks (AdHocNets), Sep. 2015, pp. 211-222.

[3] Y. Huang, W. Yu, E. Ding, and A. Garcia-Ortiz, "EPKF: energy efficient communication schemes based on Kalman filter for IoT," IEEE Internet of Things Journal, vol. 6, no. 4, pp. 6201-6211, 2019.

[4] R. Mehra, "On-line identification of linear dynamic systems with applications to Kalman filtering," IEEE Transactions on Automatic Control, vol. 16, no. 1, pp. 12-21, Feb. 1971.

[5] L. Ljung, System Identification: Theory for the User, 2nd ed., ser. Prentice Hall information and system sciences series. Prentice Hall PTR, 1999.

[6] L. Ljung, "Prediction error estimation methods," Circuits Systems and Signal Process, vol. 21, pp. 11-21, Nov.1 2002.

[7] G. C. Goodwin and K. S. Sin, Adaptive Filtering Prediction and Control, ser. Dover Books on Electrical Engineering. Dover Publications, 2009.

[8] K. S. Narendra and K. Parthasarathy, "Identification and control of dynamical systems using neural networks," IEEE Transactions on Neural Networks, vol. 1, no. 1, pp. 4-27, 1990.

[9] J. Sjöberg, Q. Zhang, L. Ljung, A. Benveniste, B. Delyon, P.-Y. Glorennec, H. Hjalmarsson, and A. Juditsky, "Nonlinear black-box modeling in system identification: a unified overview," Automatica, vol. 31, no. 12, pp. 1691-1724, 1995.

[10] K. J. Nidhil Wilfred, S. Sreeraj, B. Vijay, and V. Bagyaveereswaran, "System identification using artificial neural network," in International Conference on Circuits, Power and Computing Technologies [ICCPCT2015], 2015, pp. 1-4.

[11] M. Lutter, C. Ritter, and J. Peters, "Deep Lagrangian networks: using physics as model prior for deep learning," in International Conference on Learning Representations, 2019.

[12] H. Garnier and P. C. Young, "The advantages of directly identifying continuous-time transfer function models in practical applications," International Journal of Control, vol. 87, no. 7, pp. 1319-1338, 2014.

[13] M. Forgione and D. Piga, "Continuous-time system identification with neuralnetworks: model structures and fitting criteria," European Journal of Control, 2021.

[14] M. Schoukens and J. P. Noël, "Three benchmarks addressing open challenges in nonlinear system identification," in 20th World Congress of the International Federation of Automatic Control, Toulouse, France, Jul. 9-14 2017, pp. 448-453.

[15] M. Schoukens, P. Mattsson, T. Wigren, and J. Noël, "Cascaded tanks benchmark combining soft and hard nonlinearities," 4TU.ResearchData, Dataset. [Accessed Nov. 11, 2020].

[16] A. Janot, M. Gautier, and M. Brunot, "Data set and reference models of EMPS," in Workshop on Nonlinear System Identification Benchmarks, Eindhoven, The Netherlands, Apr.10-12 2019.

[17] M. Raissi, P. Perdikaris, and G. Karniadakis, "Physics-informed neural networks: a deep learning framework for solving forward and inverse problems involving nonlinear partial differential equations," Journal of Computational Physics, vol. 378, pp. 686-707, 2019.

[18] M. S. Lewis-Beck and A. Skalaban, "The R-squared: some straight talk," Political Analysis, vol. 2, pp. 153-171, 1990. 